\title{
Association of Fraser's syndrome and Congenital high airway obstruction syndrome (CHAOS) : case report
}

\author{
Kamal el Moussaoui ${ }^{1}$, AMINA LAKHDAR $^{2}$, and azziz baidada ${ }^{2}$ \\ ${ }^{1}$ Universite Mohammed V de Rabat Faculte de Medecine et de Pharmacie Rabat \\ ${ }^{2}$ Université Mohammed V de Rabat
}

November 20, 2020

\section{Association of Fraser's syndrome and Congenital high airway obstruction syndrome (CHAOS) : case report}

Kamal El Moussaoui ${ }^{1}$, Amina Lakhdar ${ }^{1}$, Aziz baidada ${ }^{1}$

${ }^{1}$ Department of Gynecology and Obstetrics, Maternity Souissi, University Hospital Center Ibn Sina, University Mohammed V, Rabat, Morocco

\& Corresponding author :

DR. Kamal El Moussaoui

Department of Gynecology and Obstetrics, Maternity Souissi, University Hospital Center Ibn Sina, University Mohammed V, Rabat, Morocco

Postal Adresse : immeuble 20 , n1, mahaj ryad, hay ryad, rabat-Morocco

E-mail address :dr.elmoussaouikamal.gyn.obst@gmail.com

\begin{abstract}
Congenital high airway obstruction syndrome (CHAOS) is a rare malformation that is fatal to the newborn baby after birth. It is defined as a complete or nearly complete obstruction of the upper airway. The actual incidence of UASOC is unknown. We report the case of a 24 year old primiparous patient with no medical or surgical history whose first trimester ultrasound was free of abnormalities . During the second trimester ultrasound examination showed significant subcutaneous oedema, with hyperechoic and hypertrophied lungs, hypoplastic heart and significant ascites associated with severe Oligoamnios . After having ruled out the most frequent causes: isoimmunisation (negative indirect coombs), infections (negative serologies) without being able to have a karyotype, due to the patient's refusal, the ultrasound results led us to consider the possibility of fetal hydrops secondary to the CHAOS syndrome, being the pathognomonic imaging of pulmonary hypertrophy with inversion or convexity of the diaphragms. An MRI was requested showing a probable CHAOS syndrome associated with other malformations: Laryngeal atresia , , Microphthalmia with hypertelorism and deviation of the nasal septum with lack of visualization of the thymic tissue , no clear identification of the bladder, Absence of right kidney and a hypoplastic left kidney . The evolution of the pregnancy was marked by the occurrence at the 24th week of amenorrhoea a premature delivery which gave birth to a newborn with a birth weight of $1475 \mathrm{~g}$, polymalformed with sexual ambiguity, with a distended abdomen and polydactyly. The newborn died 3 min from the post partum. Furthermore, we must mention that in this case the fetus presented Fraser's syndrome, which was not diagnosed by ultrasound . a karyotype was made showing a 46XX profile.
\end{abstract}


KEYWORDS : CHAOS; congenital high airway obstruction; Fraser's syndrome; prenatal diagnosis

\section{Case report :}

24 year old patient, primipare, without any interesting medical or surgical history. The screening ultrasound at $12+2$ weeks was without abnormalities, with a low combined risk of Down syndrome and Edwards syndrome $(<1 / 10000)$. The second trimester $(19+2$ weeks $)$ morphological ultrasound showed significant subcutaneous oedema, hyperechoic and hypertrophied lungs, hypoplastic heart and significant ascites with severe Oligoamnios.

A new control ultrasound scan at 20 weeks showed the following results: ( figure 1,2 )

a normal cranial perimeter biometry and femur length for a 20 week amenorrhoeated fetus . However, the abdominal circumference $(254 \mathrm{~mm})$ corresponded to a 29 week amenorrhoeated fetus, compared to a generalised fetal hydrops.

. At the cephalic level, it was found:

Poor visualization of the facial features due to intense oedema. the orbits were marked by microphthalmia . the nose and mouth were without obvious abnormalities.

. At the abdominal level, we found :

The presence of ascites which deformed the cavity with poor visualisation of the gastric pouch.

The left and right kidneys were not visualised, they were small and hypoplastic. Doubts about the retraction of the bladder were visualised.

. At the thoracic level it was remarkable :

The heart presented a normal anatomy, with a subjective aspect of false hypoplasia, to the detriment of the increase in the volume of the lungs.

Both lungs were elongated, hypertrophied and hyperechoic in a homogeneous way. In addition, the protrusion of the two diaphragms was towards the abdomen, with an inverted image of them, an abnormally convex image, is striking.

The trachea was larger in diameter than the outlet passages and both main bronchi were pathologically dilated.

An MRI scan was ordered at 21 weeks amenorrhoea, which was in favour of a CHAOS syndrome associated with other malformations (possible Fraser syndrome) i.e. the presence of Laryngeal Atresia, Ascites and massive fetal hydrops, Microphthalmia with hypertelorism and deviation of the nasal septum, Absence of visualization of the thymic tissue, the presence of a small gastric pouch and an unidentifiable bladder, Absence of right kidney and a hypoplastic left kidney.

A fetal hydrops was considered as the initial diagnosis. After having ruled out the most frequent causes: isoimmunisation (negative indirect Coombs), infections (negative serologies) without being able to have a karyotype due to the rejection of the patient, the ultrasound results made us think about the possibility of a fetal hydrops secondary to the CHAOS syndrome, being pathognomonic the image of a pulmonary hypertrophy with inversion or convexity of the diaphragms.

At subsequent ultrasound check-ups (23 weeks amenorrhoea), the biometry corresponded to 33 weeks amenorrhoea, with a large subcutaneous oedema, severe oligoamnios and massive ascites. The evolution of the pregnancy was marked by the occurrence of a premature delivery at 24 weeks amenorrhoea, which gave birth to a newborn with a birth weight of $1475 \mathrm{~g}$, polymalformed with sexual ambiguity, with a distended abdomen and polydactyly. ( figure 3 ) The newborn died 3 min from the post partum. A karyotype was made showing a $46 \mathrm{XX}$ profile.

\section{Discussion :}


Congenital high airway obstruction syndrome (CHAOS) is a rare malformation that is fatal to the newborn baby after birth. It is defined as a complete or nearly complete obstruction of the upper airway, which can be caused by various etiologies : laryngeal atresia, tracheal atresia or cysts or laryngeal « tissues $\gg$. Laryngeal atresia can in turn be classified as : complete laryngeal atresia (type I), subglottic obstruction (type II) or glottic obstruction (type III).

Rossi, in 1826, was the first to describe this malformation ${ }^{\mathbf{1 , 2}}$; since then, bibliographical references on this syndrome are rare, let alone references to cases corresponding to a prenatal diagnosis of the syndrome.

Between 1987 and 1994, we found 16 cases described in its prenatal form ${ }^{3}$, of which only one survived after an emergency tracheotomy at childbirth ${ }^{4}$.

In contrast, from 1995 to the present ${ }^{1-5}$, we found 14 cases, of which 5 were terminations of pregnancy (ILE) and 9 survived, although only 8 showed long-term survival.

These 2 periods allowed us to differentiate 2 phases in the knowledge of the CHAOS syndrome. The first one defines the criteria useful for diagnosis, based on the results of ultrasounds concerning structural changes secondary to atresia. The second phase describes the possible treatments and discusses their management, and also refines the diagnosis by visualising the obstructive process.

According to published literature, EXIT (Exutero intrapartum treatment) could be an important tool in the perinatal management of cases where there is strong suspicion of airway obstruction. The first successful case was described by DeCou et $\mathrm{al}^{\mathbf{6}}$, but tw as not accompanied by long-term survival. However, Crombleholme et al6 were successful in 2000.

Today, Kohl et $\mathrm{al}^{\mathbf{7}}$ have gone further by successfully channelling the obstructed area into the airways using fetoscopy. It has been observed that after the technique has been performed, the ultrasound signs secondary to the obstruction return and the pregnancy is successfully terminated.

Furthermore, we must mention that in this case the fetus presented Fraser's syndrome, which was not diagnosed by ultrasound. This makes us think that it is important to know and exclude the frequent associations of CHAOS with other morphological and genetic conditions.

More experience is needed to correctly select patients with CHAOS syndrome and on the technique itself, weighing the benefits and risks of the technique. In addition, the possibility of successfully channelling the obstructed area must be confirmed, taking into account the difficulty of channelling a severely altered and stenotic area.

Congenital high airway obstruction syndrome (CHAOS) is a frequent minor malformation, fatal intrauterine or in the immediate postpartum period. It is important to inform the patient about this pathology, its prognosis and evolution for decision making.

\section{FIGURES :}

FIGURE 1 :ULTRASOUND IMAGES : (A). Biometrics of cranial calotte with significant edema ( B) .Ascites which distorts the cavity with poor visualisation of gastric chamber

FIGURE 2: ULTRASOUND IMAGES :( C ) : Trachea with a larger diameter than the exit tracts and both main dilated bronchi(D) Normal heart, looking like a false hypoplasia, at the expense of increased lung volume.

FIGURE 3 : Post partum photo of the newborn baby with CHAOS syndrome, showing the different malformations.

Abbreviations

None

Acknowledgements 
None.

\section{Authors'contributions}

KE made substantial contributions to conception and design, acquisition of data, analysis and interpretation of data; he has been involved in drafting the manuscript and revising it critically for important intellectual content. SB made substantial contributions to interpretation of data and she has beeninvolved in drafting the manuscript and revising it critically for important intellectual content. $\mathrm{AB}, \mathrm{AL}$, and $\mathrm{AK}$ made substantial contributions to conception and design and acquisition of data; they has been involved in drafting the manuscript.

All authors read and approved the final version of the manuscript

\section{Funding}

There are no funding sources to be declared.

\section{Availability of data and materials}

The datasets used and/or analyzed during the current study are available from the corresponding author on reasonable request.

\section{Ethics approval and consent to participate}

Ethics approval has been obtained to proceed with the current study . Consent to participate not applicable.

\section{Competing interests}

The authors declare that they have no competing interests.

\section{References :}

1. Kanamori Y, Kitano Y, Hashizume K, Sugiyama M, Tomonaga T, Takayasu H, et al. A case of laryngeal atresia (Congenital High Airway Obstruction Syndrome) with chromosome 5p deletion syndrome rescued by Ex-Utero Intrapartum Treatment. J Pediatr Surg. 2004;39:25-8.

2 . Bui TH, Grunewald CH, Frenckner B, Kuylenstierna R, Dahlgren G, Edner A, et al. Successful EXIT (ex utero intrapartum treatment) procedure in a fetus diagnosed prenatally with congenital high-airway obstruction syndrome due to laringeal atresia. Eur J Pediatr Surg. 2000;10:328-33.3 . Hedrick MH, Martínez M, Filly R, Flake AW, Harrison MR, Adzick S. Congenital high airway obstruction syndrome (CHAOS): a potential for perinatal intervention. J Pediatr Surg. 1994;29:271-4.4 . Richards DS, Yancey MK, Duff P, Stieg FH. The perinatal management of severe laryngeal stenosis. Obstet Gynecol. 1992;80:537-40.5 . Kalache KD, Chaoui R, Tennstedt C, Bollman R. Prenatal diagnosis of laryngeal atresia in two cases of congenital high airway obstruction syndrome (CHAOS). Prenat Diagn. 1997;17:577-81

6. DeCou JM, Jones DC, Jacobs HD, Touloukian RJ. Successful ex utero intrapartum treatment (EXIT) procedure for congenital high airway obstruction syndrome (CHAOS) owing to laryngeal atresia. J Pediatr Surg. 1998;33:1563-5.

7. Kohl T, Hering R, Bauriedeks G, Van de Vondel P, Heep A, Keiner S, et al. Fetoscopic and ultrasoundguided decompression of the fetal trachea in a human fetus with Fraser syndrome and congenital high airway obstruction syndrome (CHAOS) from laryngeal atresia. Ultrasound Obstet Gynecol. 2006;27:84-8. 

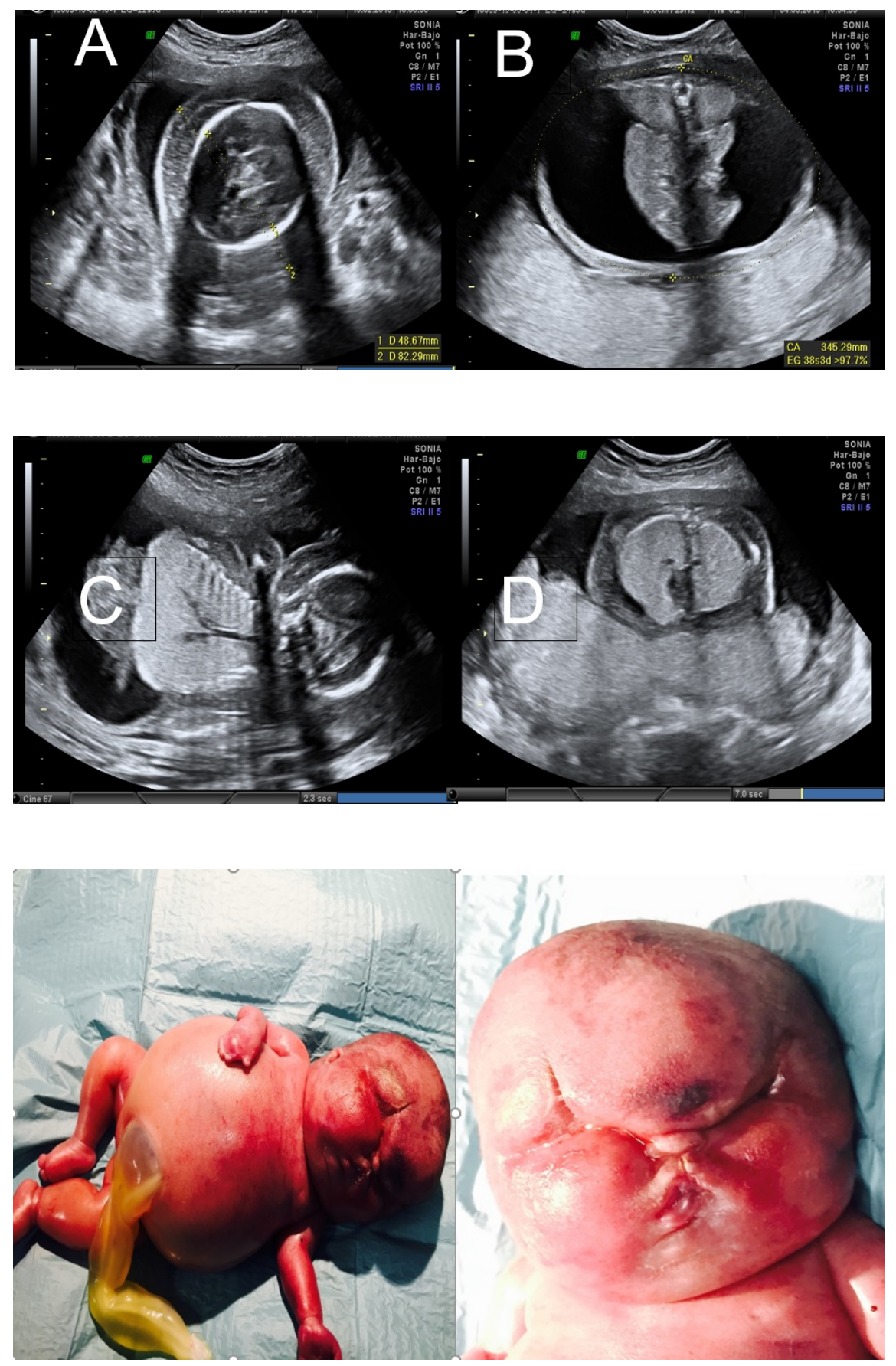